\title{
Mathematical Modeling of Magneto-Rheological Fluids in Disk-Damper for Controllable Hand-muscle Developer
}

\author{
Hao Chen ${ }^{1, a^{*}}$, Yanhong Yan ${ }^{1, \mathrm{~b}}$ and Zhaoxi Chen ${ }^{1, \mathrm{c}}$ \\ ${ }^{1}$ School of Medical Information, Guangzhou University of Chinese Medicine, China \\ achenhao@gzucm.edu.cn, byyh@gzucm.edu.cn, ${ }^{\mathrm{c} z x c h e n @ g z u c m . e d u . c n}$
}

\begin{abstract}
Keywords: Magneto-Rheological Fluids, Disk-Damper, Mathematical Modeling, Controllable Damping

Abstract. This paper presents a mathematical modeling method about magneto-rheological fluids(MRF) in disk-damper for controllable damping hand-muscle developer which used for hand rehabilitation. In our research, the characteristics of magneto-rheological fluids based on Bingham plastic model were analyzed, the formulas of transmitted torque responses were derived, and the performance of the damper were also evaluated. The results showed this kind of disc damper has a wide-range controllable torque output.
\end{abstract}

\section{Introduction}

Hands are most important organs for human beings.Hand injuries are most common illnesses. Hand rehabilitation plays an important role to improve the quality of man's life. The rehabilitation of hand dysfunction generally includes physical therapy(PT) and occupational therapy(OT). Handmuscle developer is a kind of common device for PT, but most of hand muscle developer can not be controlled its torque to fit patient's hand strength.

Magneto-rheological Fluids is a rapid growth-up intelligent material in past decade. MRF is a kind of suspension in which a mass of magnetizable micron-scaled particulate matters. When there is no magneticfield pass though it, the MRF is same as Newtonian fluids. However, when we impose a magneticfield though MRF, the suspended micron-scaled particulate matters will be magnetized, attract each others, and form a chain-liked bridge between two magnetic poles almost at the exact moment. So the macroscopic properties of the MRF convert from liquid to solid at same time. If the magnetic field is disappeared, The MRF will regain its fluidity[1]. The key parameter of MR is dynamic yield stress which is the function of the external magnetic-field intensity. Because it possesses higher yield stress $(>40 \mathrm{kpa})$ and temperature stability than electro-rheological fluid, the MRF has wilder application area.In this paper, MRF will be discussed as controllable damping media in the hand muscle developer.

\section{Mathematical Modeling of MRF}

\section{Micro-Structure Analysis of MRF}

Nowadays, researchers have made intensive studies of the micro-structure of MRF[2]. It is helpful to us to setup the structure physical model of MPF. Here we suppose that the gap between upper and bottom sliding plate is filled with MRF as showed as figure 1. When the an applied magnetic field take into effect, the suspended particulate matters in MRF form stylolitic chains whose tail-ends attach upper and bottom boards, and then the MR fluids transform into the Bingham plastics. 




Figure 1. Micro-Structure of MRF

\section{Constitutive Equation of MRF}

Due to the complexity of magnetorheological effect of MRF, there's no universally accepted or standard formula of mechanical models so far. Most of methods being used now are spline fits by experimental data[3]. For simplicity, Bingham model can be used on the premise of satisfactory accuracy.

The flow behavior of Bingham fluid is that when an applied shear stress is sufficient to exceed the yield stress, the flow will occur, and the shear stress $\tau$ has a linearly proportional to the velocity of shear deformation $r$ '. See the equation 1 below.

$$
\tau=\tau_{b}+\eta r^{\prime}
$$

\section{The Analysis of Disk-Damper}

The structure of disc damper is shown in fig 3. The MR fluid (3) is filled into the working chamber which is between rolling disc (6) and ektexine (2) of the damper.

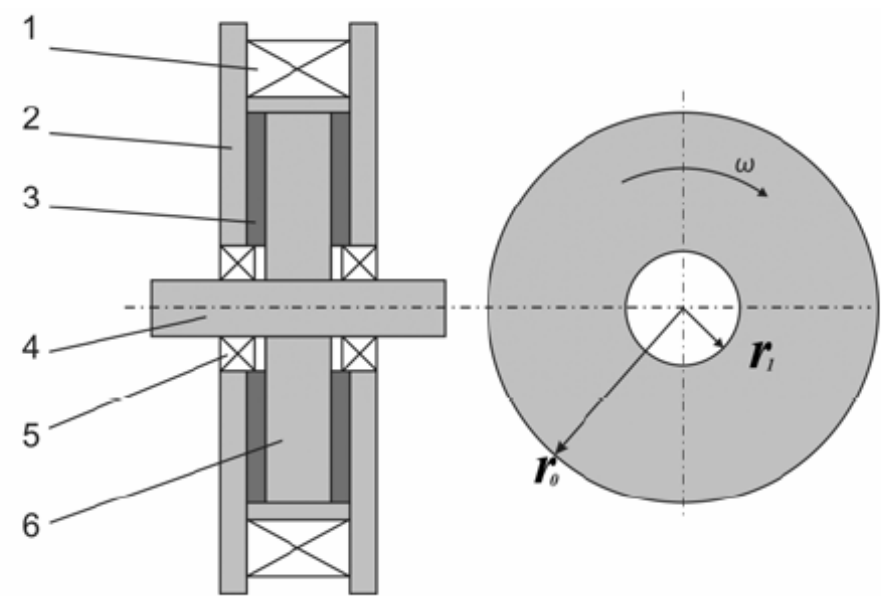

Figure 2. Structure Diagram of Disk Damper
1. Excitation Device
2. Ektexine 3.Working Chamber/MR Fluid
4. Drive Shaft
6. Rolling Disk

5. Sealed Bearing

While the rolling disk has begun to rotate due to an external rotating torque, the damper works in the shear mode. The working area is a torus whose radius ranges from $r_{0}$ to $r_{1}$. The general form of the damper's damping force may be expressed as follows[4]: 


$$
F=F_{i}+F_{m}+F_{c}
$$

Wherein formula $2, F_{i}, F_{m}, F_{c}$ is fluid damping force, electromagnetic damping force, eddy current damping force respectively.

For shear-type magneto-rheologic damper's model, the $F_{m}$, and $F_{c}$ can be expressed as follows:

$$
F_{m}=\int_{s} \frac{B_{r}^{2}}{4 \mu_{0}} d s
$$

Herein $B_{r}$ stands for the radial component of magnetic fields intensity of rolling disc, $S$ means the area of rolling disc, $\mu_{0}$ refers to permittivity.

$$
F_{c}=\frac{B^{2} t \pi r^{2}}{\rho} C_{0} r_{b}^{\prime}
$$

Herein $B$ stands for the fields intensity of rolling disc, $t$ is the thickness of the disk, $\rho$ refers to the resistance of the disk, $\mathrm{C}_{0}$ is a coefficient related with the of shape the disk.

$B_{r}$ will be considered as zero approximately when the magnetic flux is perpendicular to the rolling disk, and $F_{c}$ will be negligible also as the radius of roll disk is equal to that of magnetic field. So the fluid damping $F_{i}$ turns to be the main factor in this situation. The toque of fluid damping $T$ can be derived from formula (1) as below:

$$
T=\int_{r_{1}}^{r_{0}} 2 \pi r\left(\tau_{b}+\eta r^{\prime}\right) d r
$$

Here, $\tau_{\mathrm{b}}$ is the function of magnetic intensity $B . r$ is the velocity of shear deformation, and $\eta$ represents a constant.

As we can see from Figure 3, the flow of MRF is laminar motion and related to the working gap $h$, so $r$ ' is the function of the disc radius.

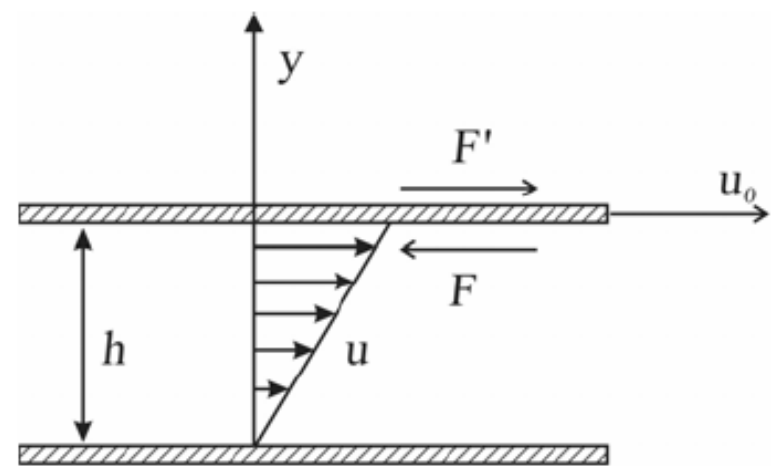

Figure 3. The distribution of shearing stress in working gap

The relationship of $r$ and $r$ ' which in any point of working face can be derived from Fig 3 showed as below.

$$
r^{\prime}=\frac{\omega r}{h}
$$

We just put formula (6) into formula (5) and infer the formula (7) as follow.

$$
T=T_{b}+T_{\eta}=\frac{2}{3} \pi \tau_{b}\left(r_{0}^{3}-r_{1}^{3}\right)+\frac{\eta \omega \pi}{2 h}\left(r_{0}^{4}-r_{1}^{4}\right)
$$

Because $T_{b}$ is in direct proportion to $B, B$ is in direct proportion to electric current $I_{s}$ which the system put in, so this part of torque belongs to the controllable damping torque.

\section{Experimental Results and It's Analysis}

After construction, we have made an experiment to confirm our formulas by using the self-made damper prototype. The experimental condition is mentioned as follows: the excitation coil has 300 of turns, and the rated speed of rolling disk is fixed at $400 \mathrm{rpm}$, the input current $I_{s}$ of excitation coil is adjustable at range of 0 to $1.0 \mathrm{~A}$. The relationship curve between damping torque and excitation 
current is shown as Fig 4. As we can see, the torque is approximately direct proportion to the current while the current exceeds a specific value.



Figure 4. The experimental curve of excitation current and damping torque

\section{Conclusion}

In this research, the mechanism of MRF damping disk was studied theoretically at first, the formula used to calculate the damping torque was derived. The curve of excitation current and damping torque was given in our experiment, and it's results indicate that the dick type damper is a good solution that fits the needs in hand muscle developer.

\section{Acknowledgment}

This research is financial supported by the Science and Technology Planning Project of Guangdong Province, China (Grant No.2011B031300015), and also supported by the National Natural Science Foundation of China (Grant No. J1310017)

\section{References}

[1] Carlson, J.D, et al, "Commercial Magneto-Rheological Fluids Devices", 5th Int. Conf.on ER Fluids, 10-14 July, 1995.

[2] PENG Xiaoqiang, YOU Weiwei, SHI Feng, "A Micro-mechanic-hydrodynamic Model for Yield Stress of Magnetorheological Fluids", Journal of National university of defense technology, Vol .28 No .4 2006,110-114.

[3] Deng Zh idang Gao Feng, et al, "State-of-Art Research on Dynamical Models of Magneto-Rheological Damper”, Journal of Vibration and Shock. Vol 25 No .3 2006,121-126.

[4] ZHU Chang-sheng, "Experimental and theoretical investigation on dynamic behavior of a flexible rotor supported on a shear-type magnetorheologic fluid damper", J.of Functional Materials, Vol.6 (37) 2006, 1006-1012. 